\title{
EXPLICIT FORMULA FOR GENERALIZATION OF POLY-BERNOULLI NUMBERS AND POLYNOMIALS WITH $a, b, c$ PARAMETERS
}

\author{
HASSAN JOLANy AND ROBERTO B. CORCINO
}

\begin{abstract}
In this paper we investigate special generalized Bernoulli polynomials with $a, b, c$ parameters that generalize classical Bernoulli numbers and polynomials. The present paper deals with some recurrence formulae for the generalization of poly-Bernoulli numbers and polynomials with $a, b, c$ parameters. Poly-Bernoulli numbers satisfy certain recurrence relationships which are used in many computations involving poly-Bernoulli numbers. Obtaining a closed formula for generalization of poly-Bernoulli numbers with $a, b, c$ parameters therefore seems to be a natural and important problem. By using the generalization of poly-Bernoulli polynomials with $a, b, c$ parameters of negative index we define symmetrized generalization of polyBernoulli polynomials with $\mathrm{a} ; \mathrm{b}$; c parameters of two variables and we prove duality property for them. Also by Stirling numbers of the second kind we will find a closed formula for them. Furthermore we generalize the Arakawa-Kaneko Zeta functions and by using the Laplace-Mellin integral, define generalization of Arakawa-Kaneko Zeta functions with $a, b, c$ parameters and obtain an interpolation formula for the generalization of poly- Bernoulli numbers and polynomials with $a, b, c$ parameters. Furthermore we present a link between this type of Zeta functions and Dirichlet series. By our interpolation formula, we will interpolate the generalization of Arakawa-Kaneko Zeta functions with $a, b, c$ parameters.
\end{abstract}

Mathematics subject classification (2010): 11B73, 11A07.

Keywords and phrases: Bernoulli numbers and polynomials, Arakawa-Kaneko Zeta functions, PolyBernoulli numbers and polynomials, generalization of Poly-Bernoulli numbers and polynomials with $a, b, c$ parameters, generalization of Arakawa-Kaneko Zeta functions with $a, b, c$ parameters.

\section{REFERENCES}

[1] D. S. Kim AND T. Kim, Higher-order Frobenius-Euler and poly-Bernoulli mixed-type polynomials, Adv. Difference Equ., 2013, 2013:251 DOI 10.1186/1687-1847-2013-251.

[2] D.S.KIM,T.KIM, Hermite and poly-Bernoulli mixed-type polynomials, Adv. Difference Equ., 2013, 2013:343 DOI 10.1186/1687-1847-2013-343.

[3] R. SANCheZ-PEREgrino, Closed formula for poly-Bernoulli numbers, Fibonacci Quart. 40 (2002), 362-364.

[4] R. Sanchez-Peregrino, A note on a closed formula for poly-Bernoulli numbers, Amer. Math. Monthly, 109, 8 (2002) 755-758.

[5] A. AdelBerg, Kummer congruences for universal Bernoulli numbers and related congruences for poly-Bernoulli numbers, Int. Math. J., 1 (2002), 53-63.

[6] C. BREWBAKER, Lonesum (0;1)-matrices and poly-Bernoulli numbers of negative index, Master's thesis, Iowa State University, 2005.

[7] M.-A. Coppo And B. CAndelpergher, The Arakawa-Kaneko zeta function, Ramanujan J. 22 (2010), 153-162.

[8] M.-S. Kim AND T. Kim, An explicit formula on the generalized Bernoulli number with order $n$, Indian J. Pure Appl. Math. 31 (2000), 1455-1461.

[9] C. BREWBAKER, A combinatorial interpretation of the poly-Bernoulli numbers and two Fermat analogues, INTEGERS, 8 (2008), A02. 
[10] K. Kamano, Sums of Products of Bernoulli Numbers, Including Poly-Bernoulli Numbers, J. Integer Seq., 13 (2010), Article 10.5.2.

[11] Y. SASAKI, On generalized poly-Bernoulli numbers and related L-functions, submitted.

[12] M. SHIKATA, Lonesum matrices and poly-Bernoulli numbers, preprint.

[13] Y. OHNO AND Y. SASAKI, On poly-Euler numbers, preprint.

[14] H. Jolany AND M. R. DARAFSHEH, Generalizations on poly-Bernoulli numbers and polynomials, International J.Math. Combin. 2 (2010), 7-14.

[15] JONAS SJÖSTRAND, Bruhat intervals as rooks on skew Ferrers boards, J. Combin. Theory Ser. A, 114 (2007) 1182-1198.

[16] S. LaUnoIS, Combinatorics of H -primes in quantum matrices, J. Algebra, 309, 1 (2007), 139-167.

[17] S. LaUnoIs, Rank $t H$-primes in quantum matrices, Comm. Algebra, 33, 3 (2005).

[18] H. K. Kim, D. S. Krotov, And J.Y. LeE, Poly-Bernoulli numbers and lonesum matrices, arXiv: $1103.4884 \mathrm{v} 1$.

[19] Q. M. Luo, F. QI AND L. Debnath, Generalization of Euler numbers and polynomials, Int. J. Math. Sci. (2003), 2003:61, 3893-3901 PII. S016117120321108X.

[20] D. W. LeE, On multiple Appell polynomials, Proc. Amer. Math. Soc., 139, 6 (2011), 2133-2141.

[21] J. Sнонат, The relation of the classical orthogonal polynomials to the polynomials of Appell, Amer. J. Math., 58 (1936), 453-464.

[22] L. Toscano, Polinomi ortogonali o reciproci di ortogonali nella classe di Appell, Le Matematica, 11 (1956), 168-174.

[23] F. A. Costabile And E. Longo, A determinantal approach to Appell polynomials, J. Comput. Appl. Math., 234, 5 (2010).

[24] M. E. H. IsmaIL, Remarks on Differential equation of Appell polynomials, J. Comput. Appl. Math., 154, 1 (2003).

[25] P. CAMERon, Poly-Bernoulli numbers and acyclic orientations, CIRCA/Algebra seminar, St Andrews, 29 January 2014.

[26] A. BAYAD AND Y. HAMAHATA, Arakawa-Kaneko L-functions and generalized poly-Bernoulli polynomials, Journal of Number Theory, 131, 6 (2011), 1020-1036. 